\title{
PARÁMETROS ELECTROCARDIOGRÁFICOS Y RADIOGRÁFICOS CARDÍACOS EN LA RAZA PERRO SIN PELO DEL PERÚ
}

\author{
Cardiac Radiographic and Electrocardiographic Parameters in the \\ Peruvian Hairless Dog
}

\author{
Lyn Carrillo V. ${ }^{1,2}$, Ricardo Grandez R. ${ }^{1,3}$, Roberto Dávila F. ${ }^{4}$
}

\section{Resumen}

El objetivo del estudio fue determinar los parámetros radiográficos cardíacos y electrocardiográficos en ejemplares de la raza Perro sin Pelo del Perú, de tamaño mediano, de la zona de Lima, Perú. Se utilizaron 30 canes de ambos sexos, adultos, con un peso corporal de 8 a $12 \mathrm{~kg}$, aparentemente sanos. Se les practicó una evaluación electrocardiográfica con seis derivaciones estándares y se analizaron las ondas ( $\mathrm{P}, \mathrm{com}$ plejo QRS y T), segmentos (PR, QRS y QT) y ritmo (sinusal normal y arritmia sinusal). La evaluación radiográfica cardíaca se realizó con tomas lateral y ventrodorsal/dorsoventral para determinar el índice cardio-vertebral (ICV) y la relación cardiotorácica (RCT). Los parámetros radiográficos cardíacos y electrocardiográficos de la raza se encontraron dentro de los valores normales para perros en general. El sexo no influyó sobre los parámetros analizados. La frecuencia de arritmia sinusal puede incrementarse a partir de los 4 años de edad.

Palabras clave: Perro sin Pelo del Perú, evaluación electrocardiográfica, radiografía cardíaca

\section{Abstract}

The objective of the study was to determine radiographic and electrocardiographic parameters in individuals of the Peruvian Hairless Dog breed, median size, in Lima, Peru. Thirty adult animals of both sexes, $8-12 \mathrm{~kg}$ body weight, and clinically healthy were used. An electrocardiographic evaluation was conducted using the six standard electrocardiographic leads and waves (P, QRS and T), segments (PR, QRS and QT) and rhythm (normal sinus and sinus arrhythmia). A radiographic evaluation was performed with lateral and ventrodorsal/dorsoventral projections to determine the cardio vertebral ratio (VHS) and total cardiac expansion (RTCE). The cardiac radiographic and

\footnotetext{
${ }^{1}$ Clínica Veterinaria Cayetano Heredia, Universidad Peruana Cayetano Heredia, Lima

${ }^{2}$ E-mail: lyn64685@yahoo.com

${ }^{3}$ E-mail: ricardo.grandez@upch.pe

${ }^{4}$ Clínica de Animales Menores, Facultad de Medicina Veterinaria, Universidad Nacional Mayor de San Marcos, Lima
} 
electrocardiographic parameters were within the values for dogs in general. Sex had no influence on the studied parameters. The frequency of sinus arrhythmia starts from 4 years of age.

Key words : Peruvian Hairless Dog, electrocardiographic evaluation, cardiac radiographic

\section{INTRODUCCIÓN}

El Perro sin Pelo del Perú (PSPP) es la raza representativa del Perú, registrada en la Federación Cinológica Internacional en 1985 (Maniero, 2004). Entre las características más saltantes está la ausencia total o parcial de pelo, debido a la presencia de un genotipo determinado por un rasgo dominante autosomal denominado displasia ectodermal (Mecklenburg, 2006).

La cardiología en caninos ha progresado con el uso de la ecocardiografía Doppler y nuevas pruebas sanguíneas para medir biomarcadores de enfermedad cardíaca (Beardow, 2008). Si bien los mencionados procedimientos facilitan el diagnóstico, su elevado costo y falta de accesibilidad hacen que el examen clínico y las pruebas radiográficas y electrocardiográficas continúen siendo las principales herramientas en el diagnóstico cardiológico. La detección temprana de soplos cardíacos, alteraciones del ritmo cardíaco, pulso yugular y otros más inespecíficos, como fatiga, intolerancia al ejercicio, congestión pulmonar o hepática, edema y ascitis, entre otros (Ware, 2006), permite un diagnóstico temprano de enfermedad cardíaca, mejorando así las probabilidades de respuesta al tratamiento y, por ende, el pronóstico del animal.

La electrocardiografía es una técnica complementaria y efectiva para detectar los diversos tipos de arritmias cardiacas. Se basa en el registro gráfico del funcionamiento eléctrico del corazón, mediante la aplicación de electrodos cutáneos que captan las diferencias de potencial producidas en el corazón y que se transmiten a través de los diferentes tejidos (Kittleson, 2000; Ynaraja y Montoya, 2005). El electrocardiograma (ECG) es un gráfico que trabaja con dos ejes, uno horizontal (que mide el tiempo en segundos) y uno vertical (que mide la amplitud de las ondas en milivoltios) (Mucha y Belerenian, 2008). El electrocardiograma normal está compuesto de una onda $\mathrm{P}$, un complejo QRS que está formado a menudo, pero no siempre, de tres ondas separadas: la onda Q, la onda $R$ y la onda $S$, y de una onda $T$ (Kittleson, 2000; Goodwin, 2005; Ynaraja y Montoya, 2005). La duración y amplitud de la onda $P$ puede ayudar a determinar la distensión de las aurículas, mientras que la distensión de los ventrículos puede ser detectada evaluando la duración, amplitud y morfología del complejo QRS. Por otro lado, se pueden observar complejos QRS de bajo voltaje en casos de efusión pericárdica, que deben ser diferenciados de voltajes bajos por obesidad (Sánchez y Venegas, 2006). La onda T no debe ser mayor del 25\% del complejo QRS. Las ondas $T$ pequeñas y bifásicas se presentan en hipopotasemia y, por el contrario, las ondas $\mathrm{T}$ altas indican hiperpotasemia.

El ECG debe ser realizado cuando se detecta arritmia cardíaca (bradicardia, taquicardia o irregularidad en el ritmo) durante la exploración física. Además, es empleado para monitorear la eficacia de la terapia antiarrítmica y determinar si la misma puede haberse desarrollado como consecuencia a la medicación (Goodwin, 2005; Sánchez y Venegas, 2006; Mucha y Belerenian, 2008). Sin embargo, se puede encontrar dificultades durante el registro del ECG debido a una serie de artefactos; por ejemplo, el jadeo del perro genera ondulaciones coincidentes con la frecuencia respiratoria en la línea base y el ronroneo del gato genera sinuosidad. Igual- 
mente, el cambio de derivación produce una línea plana durante un corto periodo que puede confundirse con parada sinusal, y el movimiento de una extremidad ocasiona desviaciones de corta duración que pueden parecer contracciones ventriculares prematuras (Kittleson, 2000). Los cambios en la posición del cuerpo y, sobre todo, la posición de las extremidades anteriores, también pueden afectar la amplitud de las ondas electrocardiográficas (MacLeod et al., 2000; Hanton y Rabemampianina, 2006).

Entre los ritmos normales en caninos se tiene el ritmo sinusal normal y la arritmia sinusal. En el primero, la frecuencia cardíaca se encuentra dentro de los parámetros normales, el ritmo es regular, la variación entre las ondas $\mathrm{R}$ es hasta $10 \%$, existen intervalos $\mathrm{P}-\mathrm{R}$ de duración constante en todas las derivaciones y tiene un complejo $\mathrm{QRS}$ por cada onda P (Montoya e Ynaraja, 2007; Mucha y Belerenian, 2008). En el caso de la arritmia sinusal, considerada normal en los perros, se produce generalmente por una variación de la intensidad de las señales de los nervios simpáticos y parasimpáticos que llegan al nódulo sinusal del corazón y alteran la frecuencia cardíaca (Guyton y Hall, 2006). El aumento de la frecuencia cardíaca durante la inspiración se produce por la activación de las fibras vagales en los pulmones, las cuales por predominio de la acción simpática inhiben el centro cardioinhibidor de la médula oblonga (Walsh y King, 2010).

Las radiografías torácicas son esenciales en la evaluación de los animales en los que se sospecha una enfermedad cardíaca (Kittleson, 2000; Nelson y Couto, 2006; Schaer, 2006). Asimismo, pueden facilitar o confirmar un diagnóstico presuntivo sobre la base de la exploración física, ayudar a establecer la severidad de la enfermedad cardíaca o recabar información sobre la eficacia de un tratamiento (Ware, 2006; Schaer, 2006). Las incidencias recomendadas para la evaluación del corazón son la látero-lateral (L-L) y dorsoventral (D-V) o ventrodorsal (V-D) (Perkins, 1979). Con la primera inci- dencia, se puede medir el índice cardio-vertebral (ICV) o cardiotorácico, medida que relaciona la dimensión cardíaca con las vértebras dorsales, cuantificando la presencia y grado de cardiomegalia (Buchanan, 2000; Kittleson, 2000; Schelling, 2005; Belerenian et al., 2007; Ford et al., 2007; Marin et al., 2007). La segunda toma (V-D o D-V) sirve para evaluar el tamaño cardíaco mediante la relación cardiotorácica $(\mathrm{RCT})$, que es la suma de las expansiones cardíacas derecha (ECD) e izquierda (ECI), y la expansión torácica total (ETT) (Belerenian et al., 2007).

El propósito del presente estudio fue determinar los parámetros radiográficos cardíacos y electrocardiográficos en ejemplares de la raza Perro sin Pelo del Perú adulto, de tamaño mediano, clínicamente normales.

\section{Materiales y Métodos}

Se utilizaron 30 perros de la raza PSPP criados en la ciudad de Lima. El estudio se llevó a cabo entre agosto a diciembre de 2009. El número mínimo de animales requerido para el estudio fue determinado por el Teorema de Límite Central (Steel y Torrie, 1992). Los criterios de inclusión para la selección de animales fueron canes entre 2 a 8 años de edad, tamaño mediano (40 a $50 \mathrm{~cm}$ a la región interescapular), peso corporal de 8 a $12 \mathrm{~kg}$, y de ambos sexos pero hembras no gestantes (FCI, 2001). Asimismo, se les practicó un examen físico general, observando mucosas, tiempo de llenado de capilar, temperatura corporal, frecuencia cardíaca y respiratoria, y auscultación cardíaca para asegurar la ausencia de soplos o algún otro tipo de alteración física (Gay et al., 2000).

Se les tomó un electrocardiograma (ECG) utilizando un electrocardiógrafo ESAOTE, modelo P80, con reguladores de velocidad $(25 \mathrm{~mm} / \mathrm{s}$ y $50 \mathrm{~mm} / \mathrm{s})$, voltaje 10 $\mathrm{mV}$ y filtros de base $0.05 \mathrm{~Hz}$, de red $60 \mathrm{~Hz}$ y $35 \mathrm{~Hz}$ de miograma. Se tomaron las derivaciones bipolares I, II y III, y las unipolares 
aVR, aVL y aVF. Para este procedimiento, el animal se colocó en la mesa de observación, sentado o de pie, sujetado por una sola persona sin empleo de tranquilizantes o anestésicos. Se colocaron electrodos tipo pinza cocodrilo en las cuatro extremidades, 2 $\mathrm{cm}$ sobre el olécranon y $4 \mathrm{~cm}$ sobre la inserción proximal del ligamento patelar. Se colocó alcohol en cada uno de los puntos de contacto electrodo-piel para facilitar la conducción eléctrica. El ECG se tomó con velocidades de 25 y $50 \mathrm{~mm} / \mathrm{s}$ durante un minuto (Montoya e Ynaraja, 2007; Atmaca y Emre, 2010).

Las placas radiográficas se tomaron con un equipo Polymobil plus (SIEMENS). Los animales fueron posicionados en la mesa de radiología con ayuda de un asistente, sin empleo de fármacos tranquilizantes. Se obtuvieron vistas latero-lateral (L-L) izquierda y dorso-ventral (D-V) o ventro-dorsal (V-D), dependiendo de la comodidad de cada paciente. Las placas se tomaron en fase de inspiración, con los valores de exposición establecidos en el protocolo radiográfico de la Clínica Veterinaria Cayetano Heredia de la Facultad de Veterinaria y Zootecnia, Universidad Peruana Cayetano Heredia. Las películas radiográficas Kodak Medical X purpose green, de tamaño $25.4 \times 30.5 \mathrm{~cm}$, se procesaron manualmente con un revelador/ reforzador y fijador/reforzador Kodak GBX. Las lecturas se realizaron en un negatoscopio convencional, determinándose el tamaño cardíaco a través del índice cardiovertebral (ICV) en la vista radiográfica latero-lateral y la relación cardiotorácica (RCT) en la vista dorso-ventral.

El ICV se calculó midiendo la longitud del eje largo del corazón y midiendo el número de vértebras torácicas que abarca, comenzando desde el extremo craneal de la cuarta vértebra torácica (T4); luego, se midió el eje corto, perpendicular al eje largo, medido en su punto de máxima extensión, calculando el número de vértebras torácicas que abarca, partiendo también de T4. El ICV es la suma del número total de vértebras torácicas (ejes largo y corto) (Buchanan y Büncheler, 1995; Kittleson, 2000; Schelling, 2005; Belerenian et al., 2007; Ford et al., 2007).

La RCT es la suma de las expansiones cardíacas derecha (ECD) e izquierda (ECI) dividida entre la expansión torácica total (ETT). Para determinar estas medidas, se traza una línea media imaginaria sobre el esternón y otra perpendicular a esta, en la base del corazón, abarcando toda la silueta cardíaca, donde la ECD es la expansión cardíaca hacia la derecha de la linea media y la ECI es la expansión cardíaca hacia la izquierda de la misma línea. Además, se traza otra línea paralela a la última, la cual abarca toda la pared torácica, constituyendo la ETT (Belerenian et al., 2007).

Se utilizó la media de los resultados obtenidos de los índices radiográficos cardíacos y electrocardiográficos como medida de tendencia central, y la desviación estándar y rango como medidas de dispersión. Asimismo, se utilizó el Chi cuadrado para evaluar la relación entre la edad ( $\geq 2$ y $<4$ años, $\geq 4$ y $<6$ años, $\geq 6$ y $\leq 8$ años), sexo (macho, hembra) y tipo de ritmo cardíaco (sinusal normal, arritmia sinusal).

\section{Resultados}

Los índices cardíacos y parámetros de la electrocardiografía se muestran en el Cuadro 1 . Se detectaron dos tipos de ritmo cardíaco. El ritmo sinusal normal se observó en 18 canes (60\%; Fig. 1) y la arritmia sinusal en 12 canes (Fig. 2). Asimismo, se encontró una asociación estadística entre presencia de arritmia sinusal con grupo etario (Cuadro 2).

\section{Discusión}

Los parámetros radiográficos y electrocardiográficos encontrados en el presente estudio fueron similares a estudios realizados en otras razas de perros (Goodwin, 
Cuadro 1. Parámetros radiográficos cardíacos y electrocardiográficos en 30 perros de la raza Perro sin Pelo del Perú, tamaño mediano, con edades de 2 a 8 años (Lima, Perú)

\begin{tabular}{|c|c|c|c|c|c|c|c|c|c|}
\hline \multirow{2}{*}{$\mathrm{ICV}^{(1)}$} & \multirow{2}{*}{$\begin{array}{r}\mathrm{RCT}^{2} \\
(\%)\end{array}$} & \multicolumn{2}{|c|}{ Onda P } & \multirow{2}{*}{$\begin{array}{c}\text { Intervalo } \\
\text { PR }\end{array}$} & \multicolumn{2}{|c|}{ Complejo QRS } & \multirow{2}{*}{$\begin{array}{c}\text { Intervalo } \\
\text { QT } \\
(\mathrm{s})\end{array}$} & \multirow{2}{*}{$\begin{array}{l}\text { Segmento } \\
\text { ST } \\
(\mathrm{mV})\end{array}$} & \multirow{2}{*}{$\begin{array}{c}\text { Onda } \\
\text { T } \\
(\mathrm{mV})\end{array}$} \\
\hline & & $\begin{array}{l}\text { Amplitud } \\
(\mathrm{mV})\end{array}$ & $\begin{array}{c}\text { Tiempo } \\
\text { (s) }\end{array}$ & & $\underset{(\mathrm{mV})}{\text { Amplitud }}$ & $\begin{array}{l}\text { Tiempo } \\
\text { (s) }\end{array}$ & & & \\
\hline 9.65 & 56 & 0.21 & 0.04 & 0.09 & 1.81 & 0.04 & 0.17 & 0.1 & 0.23 \\
\hline$(0.46)^{3}$ & (3) & $(0.04)$ & (0.004) & $(0.02)$ & $(0.48)$ & (0.004) & $(0.02)$ & $(0.02)$ & $(0.09)$ \\
\hline
\end{tabular}

1 Índice cardiovertebral

2 Relación cardiotorácica

${ }^{3}$ Desviación estándar

Cuadro 2. Relación entre grupos etarios y arritmia sinusal de 30 perros de la raza Perro sin Pelo del Perú, tamaño mediano, entre 2 y 8 años, evaluados mediante electrocardiograma

\begin{tabular}{cccc}
\hline \multirow{2}{*}{$\begin{array}{c}\text { Edad } \\
(\text { años })\end{array}$} & Total & N. ${ }^{\text {o }}$ & Arritmia sinusal \\
\cline { 3 - 4 }$\geq 2 \mathrm{a}<4$ & 17 & 3 & $17.6^{\mathrm{a}}$ \\
$\geq 4 \mathrm{a}<6$ & 5 & 3 & $60.0^{\mathrm{b}}$ \\
$\geq 6 \mathrm{a} \leq 8$ & 8 & 6 & $75.0^{\mathrm{b}}$ \\
\hline Total & 30 & 12 & 40 \\
\hline
\end{tabular}

${ }^{a, b}$ Superíndices diferentes indican diferencia estadística $(p<0.05)$

2005; Schelling, 2005; Schaer, 2006; Ware, 2006; Belerenian et al., 2007; Montoya e Ynaraja, 2007). Estos resultados permiten señalar que el síndrome de displasia ectodermal no ocasionó alteraciones anatómicas o funcionales en el corazón de canes de la raza PSPP, especialmente debido al origen mesodérmico del corazón (Mecklenburg, 2006).

La formación de la raza PSPP no ha pasado por una selección intensiva, a diferencia de otras razas como Bóxer o Yorkshire Terrier (Lamb y Boswood, 2002), que les significó modificaciones en la conformación anatómica, dando como resultado variaciones en varios índices cardíacos. Sin embargo, la sola ocurrencia de valores de los índices cardíacos en estudio dentro de los rangos de normalidad no puede asegurar la ausencia de enfermedad cardíaca; de allí que si bien, los animales del presente estudio fueron clínicamente normales, no se puede excluir la posibilidad de alguna cardiomiopatía, a pesar de que el trazado electrocardiográfico y las medidas radiográficas cardíacas se encontraron dentro de los límites normales.

Las razas braquicefálicas tienden a presentar un grado más alto de arritmia sinusal que las razas no braquicefálicas (Goodwin, 2005). El cambio morfológico de la cabeza en estos animales ha producido una mandíbula inferior de mayor longitud que la supe- 


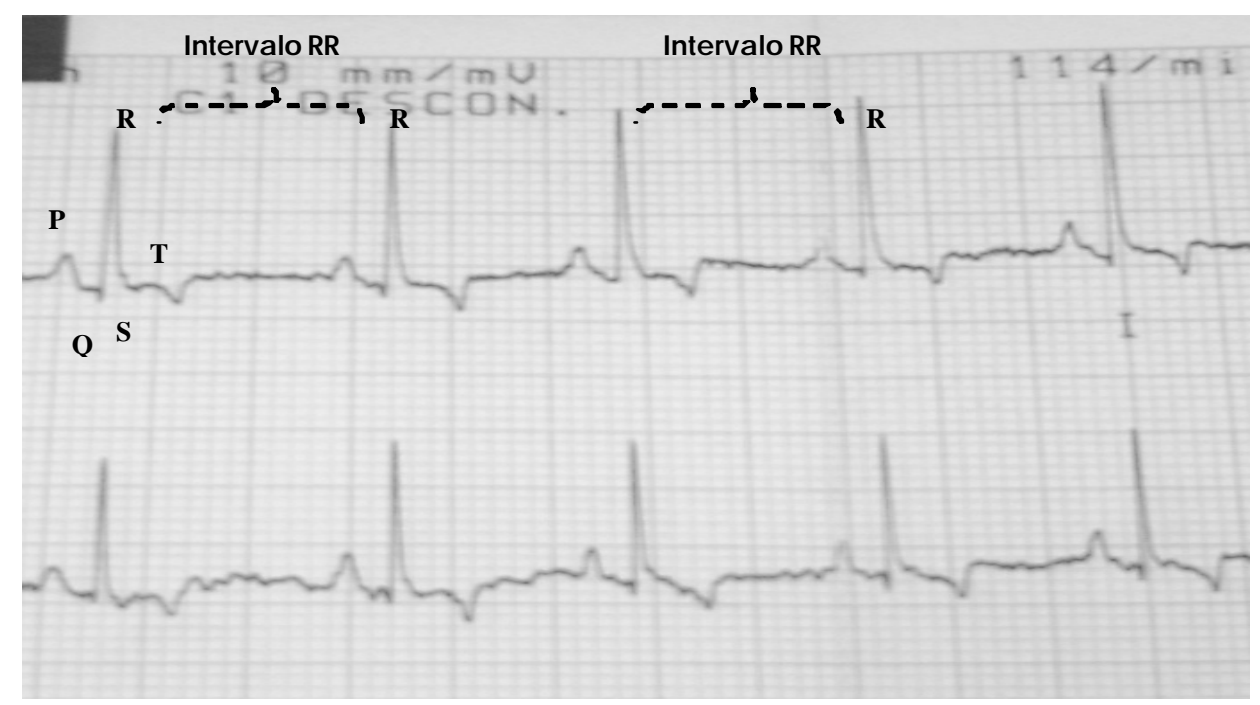

Figura 1. Ritmo sinusal regular en canino de la raza Perro sin Pelo del Perú (derivación I y II; $25 \mathrm{~mm} / \mathrm{s} ; 10 \mathrm{~mm} / \mathrm{mV}$ ). Las ondas P y los complejos QRS son normales, y los intervalos PR y RR son casi constantes

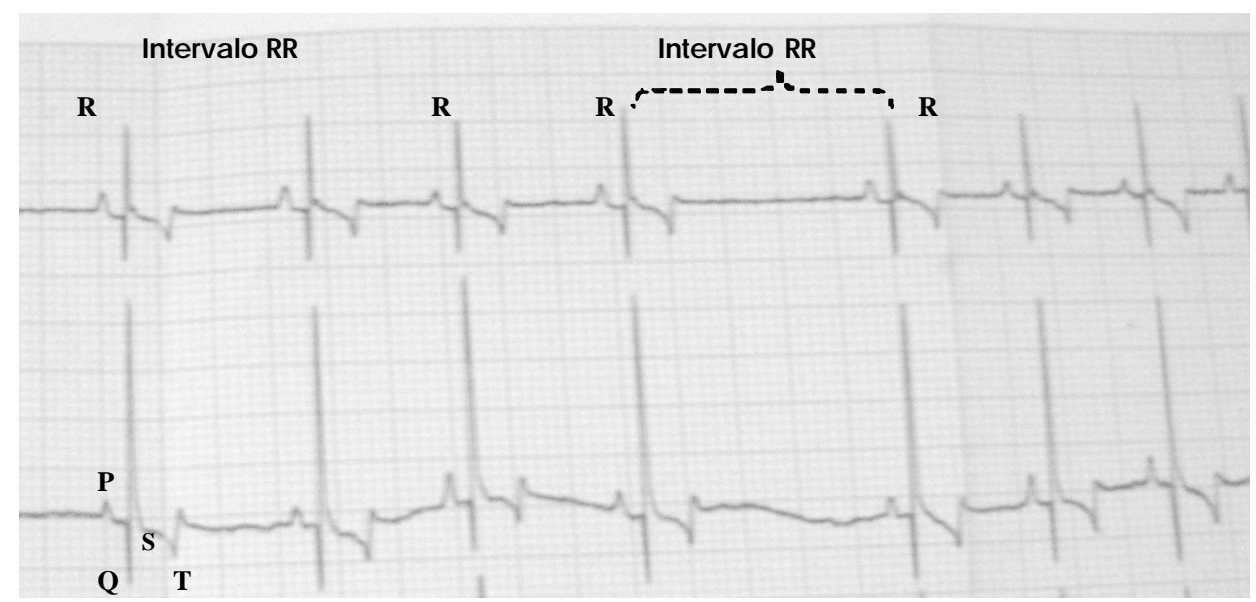

Figura 2. Arritmia sinusal respiratoria en canino de la raza Perro sin Pelo del Perú (derivación I y II; $25 \mathrm{~mm} / \mathrm{s} ; 10 \mathrm{~mm} / \mathrm{mV}$ ). Las ondas P, los complejos QRS, y los intervalos PR son normales, pero los intervalos RR son irregulares

rior, provocando estenosis nasal y elongación del paladar blando, disminuyendo de esta manera la entrada de aire a la tráquea y pulmones. El PSPP, al ser una raza mesocefálica, su nivel de arritmia sinusal no es tan marcado como en animales de razas braquicefálicas.
En la arritmia sinusal, la fluctuación de la estimulación de nervio vago durante la respiración afecta los intervalos RR (tiempo abarcado entre las ondas R) y PQ (tiempo entre el inicio de la excitación eléctrica de las aurículas e inicio de la excitación de los ventrículos) (Hanton y Rabemampianina, 
2006); es por esto que, el aumento en el tono simpático durante la inspiración acorta ambos intervalos por aumento de la frecuencia cardíaca (Montoya e Ynaraja, 2007). En el presente estudio, si bien hubo un $40 \%$ de canes con arritmia sinusal, no se alteraron los resultados electrocardiográficos pues estos ritmos cardíacos se consideran normales en el perro (Schaer, 2006; Montoya e Ynaraja, 2007; Mucha y Belerenian, 2008). El aumento significativo en la presentación de la arritmia sinusal en los canes a partir de los cuatro años de edad pudo ser debido a, que con el avance de la edad, se presentan cambios circulatorios que pueden alterar la fuerza de las señales de los nervios simpáticos y parasimpáticos que llegan al nódulo sinusal del corazón, pudiendo incrementarse la variación del tono vagal y el tono simpático, y esto ocasionaría la arritmia sinusal, sin que esto implique un tipo de condición cardíaca patológica.

El sexo no influyó de manera significativa sobre los valores del índice cardio-vertebral (ICV), corroborando los resultados de Greco et al. (2008) en otras razas; asimismo, los resultados electrocardiográficos no evidenciaron diferencias entre hembras y machos, como se demostró en estudios electrocardiográficos anteriores (Hanton y Rabemampianina, 2006).

\section{Conclusiones}

? Los parámetros radiográficos cardíacos y electrocardiográficos encontrados en la raza Perro sin Pelo del Perú se encuentran dentro de los valores establecidos para la especie canina.

? El sexo de los perros no influyó significativamente sobre los parámetros radiográficos cardíacos ni electrocardiograficos analizados.

? Se hallaron dos tipos de ritmo cardíaco normales en el electrocardiograma: ritmo sinusal normal y arritmia sinusal, don- de la proporción de animales con arritmia sinusal se incrementó a partir de los cuatro años de edad.

\section{Literatura Citada}

1. Atmaca N, Emre B. 2010. Some electrocardiographic parameters of the Kangal dogs. J Anim Vet Adv 9: 949-953.

2. Beardow A. 2008. Veterinary cardiology diagnostics. [Internet]. [18 de junio 2009]. Disponible en: http:// www.idexx.com/pubwebresources/pdf/ en_us/smallanimal/referencelaboratories/assess-cardiac-health-in-21stcentury.pdf

3. Belerenian G, Mucha C, Camacho A, Manubens Grau J. 2007. Afecciones cardiovasculares en pequeños animales. $2^{\mathrm{a}}$ ed. Argentina: Intermédica XXI. $631 \mathrm{p}$.

4. Buchanan JW, Bücheler J. 1995. Vertebral scale system to measure canine heart size in radiographs. J Am Vet Med Assoc 206:194-199.

5. Buchanan JW. 2000. Vertebral scale system to measure heart size in radiographs. Vet Clin N Am Small 30: 379-393.

6. [FCI] Fédération Cynologique Internationale. 2001. Nomenclatura de las razas. [Internet], [18 de junio 2009]. Disponible en: http://www.fci.be/

7. Ford R, Ford L, Mazaferro E, Kirk $R$, Bistner A. 2007. Urgencias en veterinaria: procedimientos y terapéutica. $8^{\mathrm{a}}$ ed. Madrid: Elsevier-Masson. 826 p.

8. Gay C, Radostits O, Mayhew I, Houston D. 2000. Clinical examination of the cardiovascular system. In: Radostits O, Mayhew I, Houston D (Eds). Veterinary clinical examination and diagnosis. USA: Harcourt Publishers. p 245-291.

9. Goodwin J. 2005. Electrocardiography. In: Tilley L, Goodwin J (eds). Manual of canine and feline cardiology. $3^{a}$ ed. Philadelphia: Saunders. p 43-70. 
10. Greco A, Meomartino L, Raiano V, Fatone G, Brunetti A. 2008. Effect of left vs right recumbency on the vertebral heart score in normal dogs. Vet Radiol Ultrasound 49: 454-455.

11. Guyton A, Hall J. 2006. Textbook of medical physiology. $11^{a}$ ed. Philadelphia: Elsevier. 1104 p.

12. Hanton G, Rabemampianina Y. 2006. The electrocardiogram of the Beagle dog: reference values and effect of sex, genetic strain, body position and heart rate. Lab Anim 40: 123-136.

13. Kittleson MD. 2000. Medicina cardiovascular de pequeños animales. Barcelona: Multimédica. $672 \mathrm{p}$.

14. Lamb C, Boswood A. 2002. Role of survey radiography in diagnosing canine cardiac disease. Comp Cont Educ Pract Vet 24: 316-326.

15. MacLeod R, Ni Q, Punske B, Ershler PR, Yilmaz B, Taccardi B. 2000. Effects of heart position on the bodysurface electrocardiogram. J Electrocardiol 33: 229-237.

16. Maniero E. 2004. Introducción a la morfología externa canina. Perú. Lima: Universidad Alas Peruanas. $586 \mathrm{p}$.

17. Marin C, Brown J, McBrien C, Baumwart R, Samii V, Couto C. 2007. Vertebral heart size in retired racing Greyhounds. Vet Radiol Ultrasound 48: 332-334.

18. Mecklenburg L. 2006. An overview on congenital alopecia in domestic animals. Vet Dermatol 17: 393-410.
19. Montoya J, Ynaraja E. 2007. Manual práctico de electrocardiografía en pequeños animales. Barcelona: ElsevierMasson. 245 p.

20. Mucha C, Belerenian G. 2008. Manual de cardiología veterinaria. Argentina: Holliday-Scott. 113 p.

21. Perkins RL. 1979. Effect of radiographic positioning on the heart shadow in dogs. Mod Vet Pract 60: 801805.

22. Sánchez M, Venegas C. 2006. Anormalidades electrocardiográficas encontradas en caninos de Bogotá, Fusagasugá y Girardot - Colombia. Red Invest Col 6: 199-205.

23. Schaer M. 2006. Medicina clínica del perro y el gato. $3^{\mathrm{a}}$ ed. Barcelona: Masson. $576 \mathrm{p}$.

24. Schelling C. 2005. Radiology of the heart. In: Tilley L, Goodwin J (eds). Manual of canine and feline cardiology. $3^{\mathrm{a}}$ ed. Philadelphia: Saunders. p 17-42.

25. Steel R, Torrie J. 1992. Bioestadística: principios y procedimientos. $2^{\mathrm{a}}$ ed. México: McGraw-Hill. 279 p.

26. Walsh S, King E. 2010. Diagnóstico por el pulso. Una guia clínica. España: Elsevier. 232 p.

27. Ware W. 2006. Enfermedades del sistema cardiovascular. En: Nelson R, Couto G (eds). Manual de medicina interna de pequeños animales. Madrid: Harcourt. p 2-221.

28. Ynaraja E, Montoya J. 2005. Manual clínico de cardiología básica en el perro y el gato. Zaragoza: Servet. 286 p. 